\title{
Neuromorphic Photonics with Laser Dynamics
}

\author{
Antonio Hurtado, Joshua Robertson, Ewan Wade \\ Institute of Photonics \\ University of Strathclyde \\ Glasgow, UK \\ antonio.hurtado@strath.ac.uk
}

\begin{abstract}
We report on the activation, inhibition and propagation of controllable neuron-like spiking signals at sub-nanosecond speeds ( $>7$ orders of magnitude faster than neurons) in artificial optical neurons based upon Vertical-Cavity Surface-Emitting Lasers. These results offer great prospects for future ultrafast photon-enabled neuromorphic computing platforms.
\end{abstract}

\section{INTRODUCTION}

Neuromorphic (brain-inspired) computing systems are receiving increasing research attention as alternative paradigms in information processing. Multiple realizations based upon micro-electronic circuitry techniques, such as Stanford's Neurogrid [1], IBM's TrueNorth [2], and the University of Manchester's SPINNAKER project [3] to name but a few have been developed yielding remarkable performances, including the generation and communication of neuron-like spikes between 1000$1,000,000$ interconnected electronic neuronal elements. However, photonic realizations for neuromorphic systems are very rapidly emerging as it is widely recognized that they can yield ultrafast speed operation whilst also helping to overcome some practical limitations of electronic-based approaches, e.g reduced bandwidths, cross talk, short communication links, etc. (for a review see [4]). These photonic approaches have been mainly based in semiconductor lasers but also on other nonlinear photonic elements such as optical modulators. These photonic neuronal models are capable of operating up to 9 orders of magnitude faster than the millisecond timescales of cortical neurons and which cannot be reached by approaches based on traditional microelectronic processors (see [4] and references therein). Among these, approaches for artificial photonic neurons based on compact Vertical Cavity Surface Emitting Lasers (VCSELs) have attracted considerable interest in recent years given their ultrasmall footprint, reduced costs, low energy consumption, ease to integrate in $2 \mathrm{D}$ arrays, potentials for high interconnectivity, and compatibility with optical communication platforms [5-11]. These features make VCSELs ideal candidates for their incorporation as artificial photonic neuronal models in future neuromorphic photonic networks.

In this work we review our work on the controllable and repeatable activation (and suppression) of neuron- like spiking dynamical signals in VCSEL-based artificial optical neuronal models. These results, similar to the operation of excitatory and inhibitory cortical neurons in the brain, are obtained at ultrafast (sub-nanosecond) speeds (up to 9 orders of magnitude faster than neurons). We will also introduce at the conference different proposed schemes both excitatory and inhibitory VCSEL-based optical neurons, based on all-optical and opto-electronic excitation techniques. Furthermore, we will also introduce our results reporting on the controllable communication between multiple spiking patterns at sub-ns rates between interconnected VCSEL-neurons under different networking arrangements. Finally, we will also introduce first results demonstrating the ultrafast optical emulation of actual neuronal circuits at ultrafast speeds using interconnected VCSEL-based artificial photonic neurons. Importantly, our results are obtained with inexpensive, off-the-shelf VCSELs operating at the key telecom wavelengths of 1310 and $1550 \mathrm{~nm}$; hence making our approach fully compatible with current optical telecommunication network technologies. These features are of great interest for future photon-enabled ultrafast neuromorphic computing platforms for novel paradigms in information processing going beyond traditional microelectronic/CMOS digital processing techniques.

\section{RESULTS}

Fig. 1 shows experimental results demonstrating the generation of controllable spiking patterns in VCSEL-neuronal models under the external optical injection of signals with added optical perturbations (or stimuli) and their successful communication to a second VCSEL-neuronal model. The encoded perturbations are configured as short power drops (with controlled temporal length and strength) encoded in the externally injected signals (see black plots in fig. 1). Full details on the experimental setup and properties of the VCSELs used in this work can be found in [6-7]. The blue plots in fig. 1 depict the responses obtained in each of the two cases at the output of a first VCSELneuronal model whilst the red plots show the responses obtained from the second connected VCSEL-neuron. In both cases, prior to the perturbation's arrival the device yields a stable temporal output as it is injectionlocked to the external injection. Then, the arrival of the incoming perturbation (providing it is strong enough) brings the system out of the locking regime and the device responds firing a spiking signal. Once the perturbation is over, the VCSEL returns back to the initial injection-locked state yielding once again a constant temporal pattern. The fired spikes have a temporal length of only approx. 100 ps with inter- 
spiking intervals under $1 \mathrm{~ns}$. We show that controllable spiking patterns with desired number of spikes can be generated, as seen in fig. 1, where two cases yielding 1 and 4 spiking events are presented. Additionally, in [67] we also demonstrated that a threshold in perturbation strength needs to be exceeded for the VCSEL-neuron to fire a sub-ns spiking event. These features are also observed in excitatory biological neurons. Importantly, the red plots in fig. 1 show that the controllably generated spiking signals from the first VCSELNeuron are successfully communicated to the second interconnected device which reproduce the exact same spiking pattern.
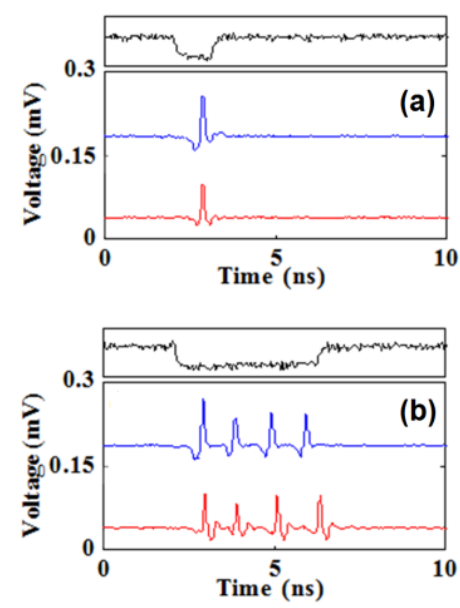

Fig. 1. Time traces measured from two connected VCSEL-neurona models (blue $\&$ red) in response to incoming signals (in black). The spiking signals generated in the first VCSEL-neuron (in blue) are injected into the second VCSEL-neuron (in red) which reproduces the same spiking pattern. Two cases are analysed with perturbations with different temporal lengths: $1.6 \mathrm{~ns}$ (top) and $4.1 \mathrm{~ns}$ (bottom).

In addition to controllably generating neuron-like spiking signals to emulate the responses of excitatory biological neurons, we have recently demonstrated the controllable suppression of spike firing outputs in VCSEL-neuronal models, much as biological inhibitory neurons do in the brain [8]. Full details on this work can be found in [8] and will be presented during the conference. Furthermore, we have also very recently demonstrated that spike firing dynamical signals at ultrafast sub-nanosecond speeds can be generated not only under all-optical experimental configurations but also under system architectures where the incoming perturbations (stimuli) are electronically-injected into the VCSEL-neuronal models via their applied bias currents [9]. Figs. 2(a-d) show examples where tuneable ultrafast spiking signals with configurable number of spikes are obtained at the output of a VCSEL-neuron under the injection of electrically-encoded perturbations with different temporal lengths. Finally, the temporal map in fig. 3 collects in a single plot the VCSEL's response to 40 consecutive perturbations for each of the four cases analyzed in figs. 2(a-d), as indicated. This map illustrates the repeatability of the generated spiking dynamics as these are consistently obtained for all consecutive perturbations entering the device.

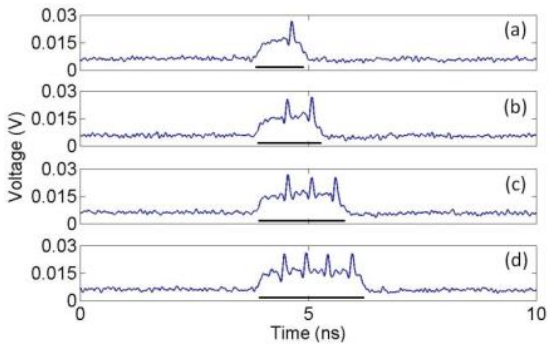

Fig. 2. Time series at the output of a VCSEL-neuronal model when an electrically-encoded stimulus with increasing temporal duration equal to: (a) $1.07 \mathrm{~ns}$, (b) $1.34 \mathrm{~ns}$, (c) $1.90 \mathrm{~ns}$ and (d) $2.35 \mathrm{~ns}$ is injected into its bias current.

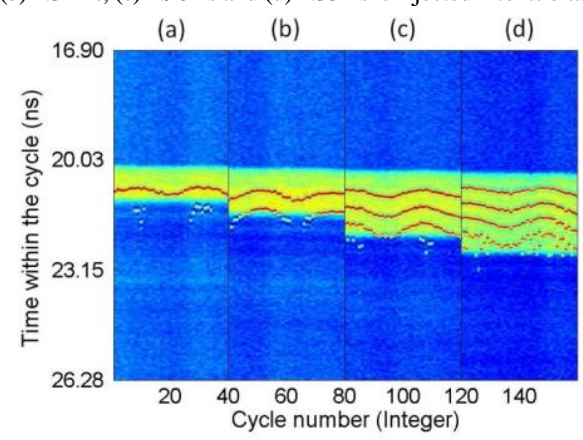

Fig. 3. Merged temporal maps of the VCSEL's output across 40 consecutive electrically-injected stimuli with increasing temporal duration: (a) $1.07 \mathrm{~ns}$, (b) $1.34 \mathrm{~ns}$, (c) $1.90 \mathrm{~ns}$ and (d) $2.35 \mathrm{~ns}$.

\section{CONCLUSION}

We review our work on VCSEL-based artificial spiking optical neurons. We show that these devices can generate (and inhibit) tuneable neuron-like spiking dynamical signals at sub-nanosecond speed rates $(>7$ orders of magnitude faster than biological neurons). We show that these ultrafast spiking regimes can be successfully communicated between interconnected VCSEL-based photonic neuronal models. During the conference we will also report on our latest results demonstrating that basic neuronal circuits can be reproduced at very high speeds using VCSEL-based neuronal models. We believe these results hold great promise for compact, highly interconnected and ultrafast optical neuronal models for future neuromorphic photonic processors going beyond current electronic computing platforms.

\section{REFERENCES}

[1] B. Benjamin et al, Proc. IEEE, 102, 699 (2014)

[2] P. A. Merolla et al, Science, 345, 668 (2014)

[3] S. Furber et al, Proc. IEEE, 102, 652 (2014)

[4] P. R. Prucnal et al, Adv. Opt. Photon., 8, 228 (2016)

[5] A. Hurtado et al, Appl. Phys. Lett. 100, 103703 (2012)

[6] A. Hurtado et al., Appl. Phys. Lett. 107, 241103 (2015)

[7] T. Deng et al., IEEE J. Quantum. Electron. 23, 1800408 (2017)

[8] J. Robertson et al., Opt. Lett. 42, 1560 (2017)

[9] J. Robertson et al, IEEE J. Sel. Top. Quantum. Electron., accepted for publication (2019)

[10]M. Turconi et al, Phys. Rev. E, 88, 022923 (2013)

[11]S. Xiang et al, IEEE J. Sel. Top. Quantum. Electron., 23, 1700207 (2017)

\section{ACKNOWLEDGEMENTS}

The authors acknowledge support from the US Office of Naval Research Global under grant ONRG - NICOP - N62909-18-1-2027. 